DOI: $10.17516 / 1999-494 X-0350$

УДК 621.391.8:535.2

\title{
Model of Formation and Propagation \\ of Infrared Radiation \\ of Heat-Radiating Aerosol Formation
}

\author{
Yuri L. Koziratsky, \\ Dmitriy V. Prohorov and Vladimir A. Baev* \\ Military Education and Research Centre of Military-Air Forces \\ «Military-Air Academy \\ Named After Professor N. E. Zhukovsky and Yu. A. Gagarin» \\ Voronezh, Russian Federation
}

Received 21.07.2021, received in revised form 10.08.2021, accepted 30.08.2021

\begin{abstract}
In the interests of substantiating the characteristics of combined aerosol curtains, a model of the processes of formation and propagation of infrared radiation of a heat-emitting aerosol formation in the atmosphere has been developed. The model makes it possible to obtain the power of the thermal radiation of active interference formed by the aerosol formation of a special aerosol-forming composition, which reaches the input of the photodetector, taking into account its energy, spatial and quantitative characteristics.
\end{abstract}

Keywords: combined interference, model, heat-emitting aerosol formation, optoelectronic means, formation of infrared radiation.

Citation: Koziratsky Yu.L., Prohorov D. V., Baev V.A. Model of formation and propagation of infrared radiation of heat-radiating aerosol formation, J. Sib. Fed. Univ. Eng. \& Technol., 2021, 14(7), 812-819. DOI: 10.17516/1999-494X-0350

(C) Siberian Federal University. All rights reserved

This work is licensed under a Creative Commons Attribution-Non Commercial 4.0 International License (CC BY-NC 4.0).

* Corresponding author E-mail address: vova1052@mail.ru 


\title{
Модель процессов формирования
}

\section{и распространения инфракрасного излучения теплоизлучающего аэрозольного образования}

\author{
Ю. Л. Козирацкий, Д. В. Прохоров, В. А. Баев \\ Военный учебно-научный центр ВВС \\ «Военно-воздушная академия \\ имени профессора Н. Е. Жуковского и Ю. А. Гагарина» \\ Российская Федерация, Воронеж
}

\begin{abstract}
Аннотация. В интересах обоснования характеристик комбинированных аэрозольных завес разработана модель процессов формирования и распространения в атмосфере инфракрасного излучения теплоизлучающего аэрозольного образования. Модель позволяет получить попадающую на вход фотоприемника мощность теплового излучения активной помехи, формируемой аэрозольным образованием специального аэрозолеобразующего состава, с учетом его энергетических, пространственных и количественных характеристик.
\end{abstract}

Ключевые слова: комбинированная помеха, модель, теплоизлучающее аэрозольное образование, оптико-электронное средство, формирование инфракрасного излучения.

Цитирование: Козирацкий, Ю. Л. Модель процессов формирования и распространения инфракрасного излучения теплоизлучающего аэрозольного образования / Ю. Л. Козирацкий, Д. В. Прохоров, В. А. Баев // Журн. Сиб. федер. унта. Техника и технологии, 2021, 14(7). С. 812-819. DOI: 10.17516/1999-494X-0350

Введение. Одним из путей повышения эффективности применения аэрозольных завес для прикрытия от средств разведки, наведения и прицеливания, функционирующих в инфракрасном (ИК) диапазоне длин волн, служит использование специальных аэрозолеобразующих химических составов $[1,2]$. Частицы аэрозоля такой завесы должны формировать тепловое излучение, являющееся активной помехой ИК-средствам, а также ослаблять оптическое излучение от прикрываемого объекта (пассивная помеха).

Актуальность. Для обоснования характеристик теплоизлучающих аэрозольных образований (ТАО) в интересах формирования эффективных активно-пассивных помех ИК-средствам разведки, наведения и прицеливания необходим специальный методический аппарат, позволяющий учесть геометрию постановки и свойства специального аэрозолеобразующего состава. Поэтому целью статьи стала разработка модели процессов формирования и распространения в атмосфере ИК-излучения теплоизлучающего аэрозольного образования, позволяющей оценить мощность теплового излучения активной помехи, формируемой аэрозольным образованием (AO) специального аэрозолеобразующего состава, с учетом его энергетических, пространственных и количественных характеристик.

Разработка модели. При моделировании следует учитывать тот факт, что теплоизлучающее аэрозольное образование будет формироваться из дискретных частиц, тепловое излучение которых будет распространяться в направлении фотоприемника (ФПр) ИК-средства, рассеиваясь и поглощаясь другими такими же частицами (рис. 1). Поэтому при рассмотрении вклада каждой частицы в формируемый $\mathrm{AO}$ поток теплового излучения помимо собственного теплового излучения частицы необходимо учитывать тепловые излучения остальных частиц аэрозольного 
образования, попадающие на данную частицу и рассеиваемые в направлении фотоприемника. Рассмотрим процессы формирования и распространения ИК-излучения такими частицами. Геометрия задачи представлена на рис. 2.

Рассмотрим в элементарном срезе $d z$ элементарный объем $d V$ (рис. 2), в котором в некоторый момент времени $t$ находится определенное число частиц с определенной температурой. При этом

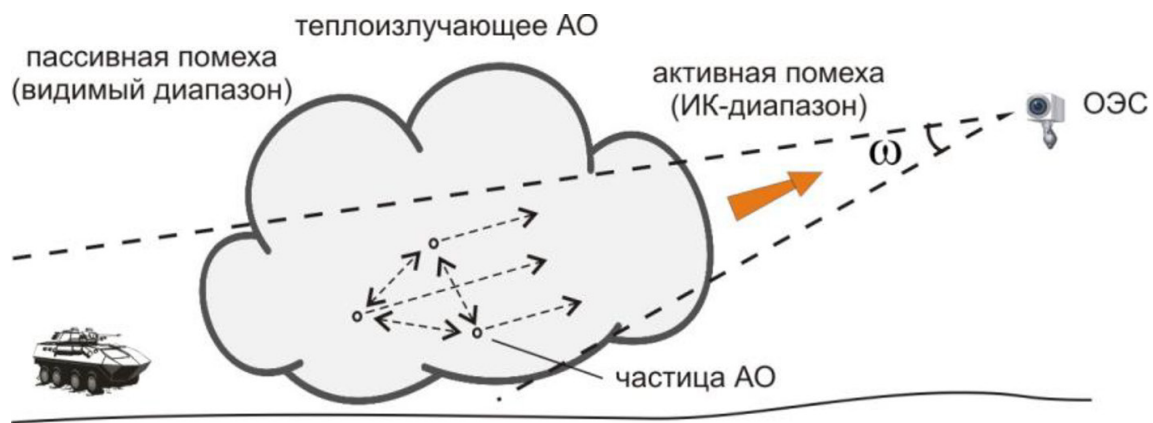

Рис. 1. К постановке задачи

Fig. 1. To the problem statement

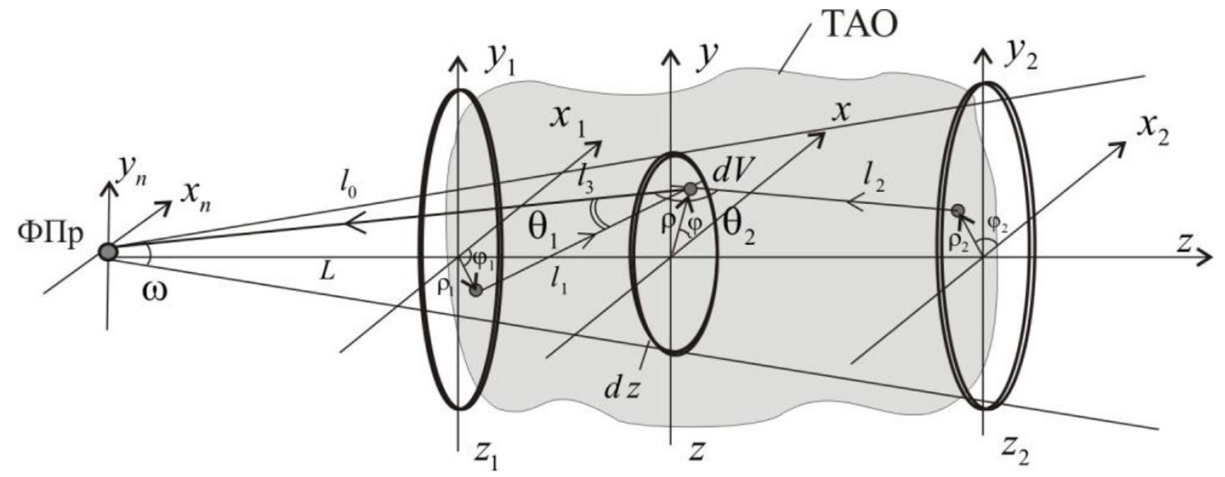

Рис. 2. Геометрия задачи

Fig. 2. Geometry of the problem

будем предполагать, что температура частиц в элементарном объеме одинакова. Тогда частицы элементарного объема $d V$ будут излучать и переизлучать электромагнитный поток, величина которого на длине волны $\lambda$ будет характеризоваться спектральными коэффициентом излучения $\varepsilon_{\lambda}$, показателем поглощения $k_{n \lambda}$ и показателем рассеяния $\sigma_{\lambda}$ этого объема.

На рассматриваемый элементарный объем попадает излучение от всех других элементарных объемов, которое рассеивается в соответствии с показателем $\sigma_{\lambda}$ и будет суммироваться с собственным излучением частиц объема.

Величина собственной спектральной плотности мощности теплового излучения от элементарного объема $d V=\rho d \rho d \varphi d z$ ТАО на входе фотоприемника будет определяться выражением [3] 


$$
d P_{0 \lambda}=\frac{k_{n \lambda} \varepsilon_{\lambda}}{\pi\left(l_{0}+l_{3}\right)^{2}} M_{\lambda}^{0}(T) e^{-\alpha_{1 \lambda} l_{3}} e^{-\alpha_{0 \lambda} l_{0}} d V d x_{n} d y_{n}
$$

где $\alpha_{0 \lambda}$ и $\alpha_{1 \lambda}=\sigma_{\lambda}+k_{n \lambda}$ - суммарные спектральные показатели ослабления оптического излучения на трассе распространения вне аэрозольного образования и внутри него соответственно $\left[M_{-1}\right] ; M_{\lambda}^{o}(T)=c_{1} \cdot \lambda^{-5} \cdot\left[\mathrm{e}^{c_{2} / \lambda T}-1\right]^{-1}-$ спектральная плотность энергетической светимости абсолютно черного тела с абсолютной температурой $T ; c_{1}$ и $c_{2}$ - постоянные Планка; $l_{3}$ и $l_{0}$ - расстояния от элементарного объема до точки пересечения линии визирования «ФПр - элементарный объем» с условной границей раздела сред ТАО и атмосферы и от точки пересечения до ФПр соответственно.

Для полидисперсной среды с целью учета концентрации и размеров частиц в элементарном объеме можно использовать следующие выражения для определения объемных спектральных коэффициентов рассеяния и поглощения соответственно:

$$
\sigma_{\lambda}=N \int_{0}^{\infty} \sigma_{u \lambda}(a) f(a) d a ; k_{n \lambda}=N \int_{0}^{\infty} k_{n u \lambda}(a) f(a) d a,
$$

где $N$ - количество частиц в единице объема; $k_{n ч \lambda}(a), \sigma_{\text {чл }}(a)$ - коэффициенты поглощения и рассеяния на частице радиусом $a ; f(a)$ - функция, описывающая распределение размеров частиц.

Для удобства дальнейшей работы с полученным выражением (1) запишем его для случая единичного объема $d V$ и единичной площади ФПр в виде

$$
P_{0 \lambda}=\frac{k_{n \lambda} \varepsilon_{\lambda}}{\pi\left(l_{0}+l_{3}\right)^{2}} M_{\lambda}^{0}(T) e^{-\alpha_{1 \lambda} l_{3}} e^{-\alpha_{0} l_{0}}
$$

Определим величину потока рассеянной составляющей, попадающей на вход ФПр $[4$, 5]. На элементарный объем, находящийся на удалении $z$, будет попадать излучение от всех элементарных объемов ТАО, находящихся как на удалении $z_{1}<z$, так и на удалении $z_{2}>z$ (рис. 2) [6]. Тогда спектральная плотность мощности излучения элементарного объема, находящегося на удалении $z_{1}\left(z_{2}\right)$ от ФПр, рассеянного объемом $d V$, расположенным на удалении $z$, и попадающего на вход ФПр, может быть определена

$$
\begin{aligned}
& d P_{-\lambda}=\frac{k_{n \lambda} \varepsilon_{\lambda} \sigma_{\lambda} f_{\lambda}\left(\theta_{1}\right)}{4 \pi^{2} l_{1}^{2}\left(l_{0}+l_{3}\right)^{2}} M_{\lambda}^{o}(T) e^{-\alpha_{1 \lambda}\left(l_{1}+l_{3}\right)} e^{-\alpha_{0} l_{0}} \rho_{1} d \rho_{1} d \varphi_{1} d z_{1} d V d x_{n} d y_{n} ; \\
& d P_{+\lambda}=\frac{k_{n \lambda} \varepsilon_{\lambda} \sigma_{\lambda} f_{\lambda}\left(\theta_{2}\right)}{4 \pi^{2} l_{2}^{2}\left(l_{0}+l_{3}\right)^{2}} M_{\lambda}^{o}(T) e^{-\alpha_{1 \lambda}\left(l_{2}+l_{3}\right)} e^{-\alpha_{0} l_{0}} \rho_{2} d \rho_{2} d \varphi_{2} d z_{2} d V d x_{n} d y_{n},
\end{aligned}
$$

где $f_{\lambda}(\theta)=\frac{\sigma_{\lambda}(\theta)}{\sigma_{\lambda}}$ - спектральная индикатриса рассеяния; $l_{1}$ и $l_{2}-$ расстояния от элементарных объемов, находящихся на удалении $z_{1}$ и $z_{2}$ от кромки ТАО соответственно до объема $d V$.

Для единичного объема $d V$ и единичной площади ФПр выражения (4) и (5) запишем в виде

$$
\begin{gathered}
d P_{-\lambda}^{\prime}=\frac{k_{n \lambda} \varepsilon_{\lambda} \sigma_{\lambda} f_{\lambda}\left(\theta_{1}\right)}{4 \pi^{2} l_{1}^{2}\left(l_{0}+l_{3}\right)^{2}} M_{\lambda}^{o}(T) e^{-\alpha_{1 \lambda}\left(l_{1}+l_{3}\right)} e^{-\alpha_{02} l_{0}} \rho_{1} d \rho_{1} d \varphi_{1} d z_{1} ; \\
d P_{+\lambda}^{\prime}=\frac{k_{n \lambda} \varepsilon_{\lambda} \sigma_{\lambda} f_{\lambda}\left(\theta_{2}\right)}{4 \pi^{2} l_{2}^{2}\left(l_{0}+l_{3}\right)^{2}} M_{\lambda}^{o}(T) e^{-\alpha_{1 \lambda}\left(l_{2}+l_{3}\right)} e^{-\alpha_{0 \lambda} l_{0}} \rho_{2} d \rho_{2} d \varphi_{2} d z_{2} . \\
-815-
\end{gathered}
$$


Принимаемая ФПр спектральная плотность мощности излучения всех элементарных срезов ТАО слева (справа) от элементарного объема $d V$, рассеянного этим элементарным объемом в направлении ФПр, может быть записана в виде выражений

$$
\begin{aligned}
& d P_{-\lambda}^{\prime \prime}=\int_{L}^{z-\Lambda z} \int_{0}^{2 \pi} \int_{0}^{D_{a}\left(z_{1}\right)} d P_{-\lambda}^{\prime} ; \\
& d P_{+\lambda}^{\prime \prime}=\int_{z}^{L+L_{a}} \int_{0}^{2 \pi} \int_{0}^{D_{a}\left(z_{2}\right)} d P_{+\lambda}^{\prime},
\end{aligned}
$$

где $L_{a}$ - толщина ТАО; $D_{a}-$ высота ТАО.

С учетом полученных выражений (1), (8) и (9) определим суммарную спектральную плотность мощности излучения от единичного элементарного объема $d V$ TАО на входе фотоприемника с единичной площадью апертуры

$$
d P_{\Pi \lambda}=d P_{+\lambda}^{\prime \prime}+d P_{-\lambda}^{\prime \prime}+P_{0 \lambda}
$$

Для определения мощности теплового излучения всего ТАО, принимаемого фотоприемником с апертурой $S$, необходимо решить интегральное уравнение

$$
P_{\Pi \sigma x}=\int_{\lambda_{1}}^{\lambda_{2}} \iint_{(S)} \iiint_{(V)} d P_{\Pi \lambda} \rho d \rho d \varphi d z d x_{n} d y_{n} d \lambda,
$$

где $\lambda_{1} \ldots \lambda_{2}=\Delta \lambda$ - рабочий спектральный диапазон ИК-средства; $V$ - объем ТАО в поле зрения ИК-средства.

С учетом геометрии формируемого полем зрения ИК-средства объема ТАО выражение (11) можно записать в виде

$$
P_{\Pi I \varepsilon x}=\int_{\lambda_{1}}^{\lambda_{2}} \iint_{S} \int_{L}^{L+L_{a}} \int_{0}^{2 \pi} \int_{0}^{z \cdot \operatorname{tg}\left(\arccos \left(1-\frac{\omega}{2 \pi}\right)\right)} d P_{\Pi \lambda} \rho d \rho d \varphi d z d x_{n} d y_{n} d \lambda .
$$

Для решения расчетных задач в соответствии с полученными выражениями достаточно выразить переменные $l_{0}, l_{1}, l_{2}, l_{3}, \theta_{1}, \theta_{2}$ через переменные интегрирования и известные параметры.

Так, в системе координат фотоприемника при условии, что точка приема в начале его системы координат, можно получить

$$
\begin{aligned}
& l_{1}=\sqrt{\left(\rho \cos \varphi-\rho_{1} \cos \varphi_{1}\right)^{2}+\left(\rho \sin \varphi-\rho_{1} \sin \varphi_{1}\right)^{2}+\left(z-z_{1}\right)^{2}} ; \\
& l_{2}=\sqrt{\left(\rho \cos \varphi-\rho_{2} \cos \varphi_{2}\right)^{2}+\left(\rho \sin \varphi-\rho_{2} \sin \varphi_{2}\right)^{2}+\left(z-z_{2}\right)^{2}} \\
& l_{3}=\frac{z-L}{\cos \left(\operatorname{arctg} \frac{\rho}{z}\right)} ; l_{0}=\frac{L}{\cos \left(\operatorname{arctg} \frac{\rho}{z}\right)} ;
\end{aligned}
$$

с учетом замены переменных $x=\rho \cdot \cos \varphi, y=\rho \cdot \sin \varphi$

$$
\begin{gathered}
\theta_{1}=180^{\circ}-\arccos \frac{\left|\left(x-x_{1}\right) x+\left(y-y_{1}\right) y+\left(z-z_{1}\right) z\right|}{\sqrt{\left(x-x_{1}\right)^{2}+\left(y-y_{1}\right)^{2}+\left(z-z_{1}\right)^{2}} \cdot \sqrt{x^{2}+y^{2}+z^{2}}} \\
-816-
\end{gathered}
$$




$$
\theta_{2}=\arccos \frac{\left|\left(x-x_{2}\right) x+\left(y-y_{2}\right) y+\left(z-z_{2}\right) z\right|}{\sqrt{\left(x-x_{2}\right)^{2}+\left(y-y_{2}\right)^{2}+\left(z-z_{2}\right)^{2}} \cdot \sqrt{x^{2}+y^{2}+z^{2}}},
$$

где $L$ - минимальное расстояние от приемника до аэрозольного образования.

На рис. 3 представлены результаты расчетов для типовых условий и следующих исходных данных: рабочий спектральный диапазон ФПр 3-5 мкм; радиус формирующей оптики ФПр 0,03 м; размеры ТАО $30 \times 30 \times 30 \mathrm{м} ; L=1$ км; $T=330 \mathrm{~K} ; \varepsilon_{\lambda}=0,8 ; k_{n \lambda}=10^{-3} \mathrm{M}^{-1}$. Влияние АО как пассивной помехи принято характеризовать коэффициентом пропускания АО $T_{a}(\sigma)$ [2], который в зависимости от его рассеивающих свойств для указанных исходных данных может принимать значения, представленные на рис. 4.

Результаты анализа полученных зависимостей показывают, что увеличение рассеивающих свойств АО приводит к уменьшению активной помеховой составляющей комбинирован-

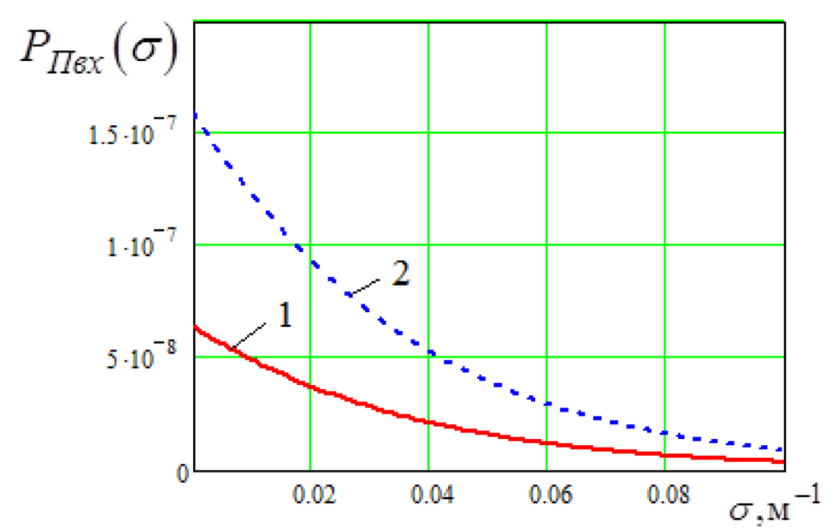

Рис. 3. Зависимости мощности теплового излучения АО на входе фотоприемника от показателя рассеяния его частиц при различных значениях поля зрения фотоприемника: $1-\omega=2 \cdot 10^{-4} \mathrm{cp} ; 2-\omega=5 \cdot 10^{-4} \mathrm{cp}$

Fig. 3. Dependences of the power of thermal radiation of aerosol formation at the input of the photodetector on the scattering index of its particles at different values of the field of view of the photodetector: $1-\omega=2 \cdot 10^{-4} \mathrm{sr}$; $2-\omega=5 \cdot 10^{-4} \mathrm{sr}$

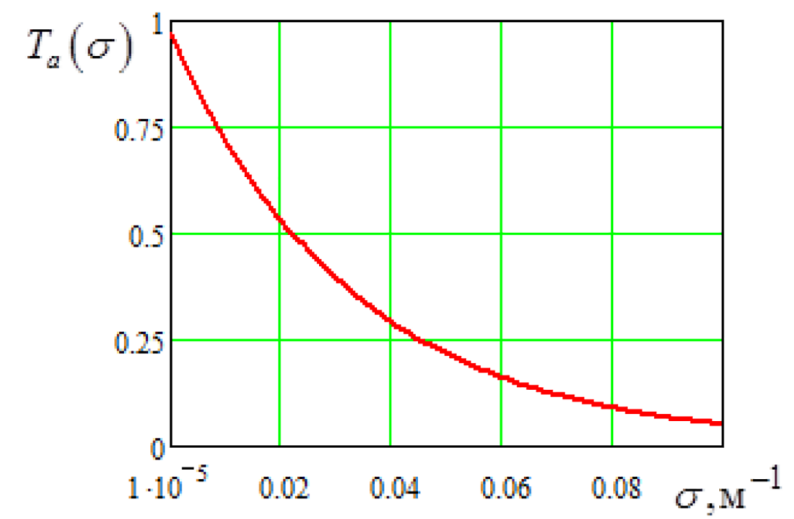

Рис. 4. Зависимость коэффициента пропускания АО от показателя рассеяния его частиц

Fig. 4. Dependence of the transmittance of aerosol formation on the scattering index of its particles 
ной помехи при одновременном увеличении маскирующего эффекта за счет пассивной помехи [2], что следует учитывать при решении задач по формированию аэрозолеобразующего состава TAO.

Выводы. Таким образом, разработана модель процессов формирования и распространения в атмосфере ИК-излучения теплоизлучающего аэрозольного образования, позволяющая обосновывать характеристики комбинированных аэрозольных завес в интересах повышения эффективности их применения. На основе введения элементарных объемов и элементарных срезов, включающих элементарные объемы, и использования основных законов образования, поглощения и рассеяния теплового излучения получена система интегро-дифференциальных уравнений, описывающих процедуры формирования ИК-излучения элементарным объемом, молекулярное и аэрозольное (на частицах) рассеяние падающего на рассматриваемый элементарный объем ИК-излучения с других объемов теплоизлучающего аэрозольного образования, переноса прямого (образованного) и рассеянного ИК-излучения от всех элементарных объемов, попадающих на вход приемника ИК-средства.

\section{Список литературы / References}

[1] Юхно П.М. Преднамеренные оптические помехи высокоточному оружию. М.: Радиотехника, 2017. 640 с. [Yukhno P. M. Deliberate optical interference with precision weapons. Moscow: Radio engineering, 2017. 640 p. (in Russian)]

[2] Козирацкий Ю.Л., Козирацкий А.Ю., Прохоров Д.В. Модели пространственного и частотного поиска. М.: Радиотехника, 2013. 344 с. [Koziratsky Yu.L., Koziratsky A. Yu., Prohorov D.V. Models of spatial and frequency search. Moscow: Radiotekhnika, 2013. 344 p. (in Russian)]

[3] Мирошников М.М. Теоретические основы оптико-электронных приборов. Л.: Мишиностроение, 1977. 696 с. [Miroshnikov M. М. Theoretical foundations of optoelectronic devices. Leningrad: Mishinostroenie, 1977. 696 p. (in Russian)]

[4] Козирацкий А.Ю., Прохоров Д.В., Шамарин А.В. Модель поиска цели оптикоэлектронными средствами в условиях комбинированных помех, формируемых на основе подсвета аэрозольного облака оптическим излучением, ВИРЭ, Вестник Военного института радиоэлектроники, 2005, 3(1), 23-35 [Koziratsky A. Yu., Prohorov D. V., Shamarin A. V. Model of target search by optoelectronic means in conditions of combined interference formed on the basis of illumination of an aerosol cloud with optical radiation, VIRE, Bulletin of the Military Institute of Radio Electronics, 2005, 3(1), 23-35 (in Russian)]

[5] Козирацкий А.Ю., Прохоров Д. В. Методика оценки возможности функционирования лазерной линии связи на основе использования аэрозольных образований с целью обхода препятствий на трассе распространения, Телекоммуникации, 2006, 8, 33-36 [Koziratskiy A. Yu., Prohorov D. V. Methodology for assessing the possibility of the functioning of a laser communication line based on the use of aerosol formations in order to avoid obstacles along the propagation path, Telecommunications, 2006, 8, 33-36 (in Russian)]

[6] Козирацкий Ю.Л., Прохоров Д.В., Плеве В.В., Хроликов В.Е. Модель процессов формирования и переноса широкополосного ИК-излучения от факела твердотопливного двигателя ЗУР до тепловизионного средства разведки, Радиотехника, 2011, 8, 12-16 [Koziratsky Yu.L., 
Prohorov D. V., Plehve V.V., Khrolikov V.E. Model of the processes of formation and transfer of broadband infrared radiation from the torch of a solid-propellant anti-aircraft guided missile engine to a thermal imaging reconnaissance tool, Radiotekhnika, 2011, 8, 12-16 (in Russian)] 